\title{
ATMOSPHERIC INTERNAL GRAVITY WAVES AS A SOURCE \\ OF QUASIPERIODIC VARIATIONS OF THE COSMIC RAY \\ SECONDARY COMPONENT AND THEIR \\ LIKELY SOLAR ORIGIN*
}

\author{
(Invited Review) \\ A. M. GALPER, V. G. KIRILLOV-UGRYUMOV, N. G. LEIKOV, and B. I. \\ LUCHKOV
}

The Moscow Physical Engineering Institute, 115409. Moscow

\begin{abstract}
Hard gamma-radiation fluctuations with the periods from 4 to 60 min were investigated in the course of balloon flights at altitudes of $30-40 \mathrm{~km}$. Quasiperiodic intensity variations (QPV) were observed with periods of $5 \mathrm{~min}, 12-15 \mathrm{~min}$, and 23-26 min, those of $5 \mathrm{~min}$ predominating. QPV last no longer than several hours, their associated amplitudes ranging from 5 to $20 \%$. QPV were observed both in mid-latitudes and in the tropics, their detection probability for $3^{\mathrm{h}}$ exposure being 0.3 . In the total charged component QPV with comparable amplitudes were not registered. Synchronous atmospheric pressure variations were recorded practically with an amplitude 20 times less than that of gamma-radiation. This suggest short internal gravity waves (IGW) in the stratosphere in the range from 10 to $100 \mathrm{~km}$ as the most likable source of QPV. Since the temperature profile of the Earth atmosphere provides conditions for superdistant waveguiding propagation of short IGW with a period of $\sim 5 \mathrm{~min}$ at altitudes of 110 and $30 \mathrm{~km}$, the source of waves can be well away from the point of their registration. The IGW generation in the stratosphere can be attributed to the resonance caused by global solar oscillations with low /modes. The resonance probability is likely to be due to the hard solar radiation variations which are absorbed in the ozone layer. The coincidence of the frequency oscillation range in the chromosphere and that of IGW in the stratosphere suggests an IGW resonant excitation mechanism in the Sun-Earth system.
\end{abstract}

The observations at $46^{\circ} \mathrm{N}$ geomagnetic latitude (cutoff rigidity $R=3.5 \mathrm{GV}$ ) and $8^{\circ} \mathrm{N}$ geomagnetic latitude $(R=16.9 \mathrm{GV})$ by high altitude balloons in 1972-79 recorded quasiperiodic gamma-ray intensity variations (QPV) with an energy of more than $40 \mathrm{MeV}$ (Galper et al.) Measurements made with a spark chamber telescope, with a geometrical factor of $115 \mathrm{~cm}^{2}$ ster and an effective aperture of $40^{\circ}$ (Galper et al., 1974) were carried out at altitudes from 4 to $10 \mathrm{~g} \mathrm{~cm}^{-2}$ of the residual atmosphere, the measuring time lasting from 5 to $10 \mathrm{hr}$.

Simultaneously, the intensity of charged particles was recorded by means of a single counter (the threshold energy of $\sim 1 \mathrm{MeV}$ ) and a directed counter telescope detecting electrons with energies of more than $60 \mathrm{MeV}$ and protons with energies of more than $300 \mathrm{MeV}$. The atmospheric pressure was also recorded, a sensor with a precision of $10^{-2}$ mbar being used in one of the balloon flights.

Time-variation analysis made in the frequency range from $3 \times 10^{-4}$ to $4 \times 10^{-3} \mathrm{~Hz}$ (the associated periods from 4 to $60 \mathrm{~min}$ ) revealed the following regularities of QPV that might suggest their origin:

* Proceedings of the 66th IAU Colloquium: Problems in Solar and Stellar Oscillations, held at the Crimean Astrophysical Observatory, U.S.S.R., 1-5 September, 1981. 
(1) QPV with the periods of $5 \mathrm{~min}, 12-15 \mathrm{~min}$, and 23-26 min are observed, those of 5 min predominating;

(2) QPV last no longer than several hours, their associated amplitudes ranging from 5 to $20 \%$;

(3) the QPV detection probability for $3 \mathrm{hr}$ exposure is about 0.3 ;

(4) there are no variations in the charged component, at least with the amplitudes more than $5 \%$;

(5) atmospheric pressure variations were recorded which are prectically synchronous with QPV of gamma-radiation, but with amplitudes smaller by a factor of 20 .

It is possible to explain the observed QPV data. Hard gamma-radiation in the stratosphere is likely to be due to the interaction of primary cosmic rays with the matter of the atmosphere. Gamma-ray intensity can be expressed as: $I_{\gamma}=A I_{C R}(>R) t$, where $I_{C R}(>R)$ is the primary cosmic ray intensity with an energy higher than the local cutoff rigidity $R, t=\int_{z_{0}}^{\infty} \rho(z) \mathrm{d} z$ is the thickness of the residual atmosphere, $\rho(z)$ is air density, and $z_{0}$ is the balloon altitude. Since the air density changes exponentionally as $\rho(z) \sim e^{-z / H}$, only the thickness of the atmosphere comparable to the standard height $H=7 \mathrm{~km}$ is essential. The interpretation of QPV in terms of $I_{C R}$ or $R$ variations seems unlikely since no marked variations in the charged component are recorded. In view of modern ideas of internal gravity waves (IGW) it is, however, possible to interpret the obtained data in terms of thickness $t$ variations.

IGW are exhibited in periodic variations of atmospheric parameters, air density in particular (Gossard and Hook, 1978; Hines, 1960). These variations would, in fact, amount to $t$ variations. The representation of an isothermal atmosphere where IGW behave as flat waves will hold as the first approximation. Short IGW with wavelengths of about $100 \mathrm{~km}$ are essentially transversal (Golitzin, 1965) and their relative pressure fluctuations are much smaller than those of density and temperature. The approximated dispersion ratio is $\omega=\omega_{\mathrm{B}} \cos \alpha$, where $\alpha$ is the phase velocity horizontal angle, and $\omega_{\mathrm{B}}$ is the Brunt frequency (Grossard and Hook, 1978). The corresponding $B$-period at balloon altitudes is $4.8 \mathrm{~min}$ in mid-latitudes and $4.5 \mathrm{~min}$ in the tropics.

Variability of $t$, and hence of $I$, will be maximum in the direction along the wave phase plane. Therefore, if the gamma-telescope axis orients to the zenith, as it did in our case, the observation would give predominently QPV due to horizontal IGW with a period of about $5 \mathrm{~min}$.

The ratio $\Delta \rho / \rho \sim \sqrt{\rho}$ holds for the isothermal atmosphere, from where gamma-ray intensity has to change as $\Delta I_{\gamma} / I_{\gamma}=\Delta t / t=\beta(\Delta \rho / \rho)_{z_{0}}$, where $\beta=2$, which implies that QPV relative amplitude is twice as high as that of density variations at altitude $z_{0}$. For the real atmosphere $I<\beta<2$ since short IGW undergo scattering at the altitudes between 30 and $50 \mathrm{~km}$ (and at altitude of $110 \mathrm{~km}$ ) due to a high temperature gradient involved. This effect reduces QPV amplitude and at the same time provides conditions for superdistant waveguiding propagation of short IGW (Dikii, 1969) and, hence, increases the chance of their observation.

The fact that no marked variations of the charged component intensity $\left(I_{c}\right)$ have been recorded seems natural in the frame of IGW explanation because of a weak altitude 
dependence of $I_{c}(z)$ which makes that $\Delta I_{c} / I_{c} \ll \Delta t / t$. Some modulation effects due to IGW must, however, be observable in the secondary charged component which is really the case (Komoda et al., 1975).

The QPV-IGW association may suggest an explanation for the origin of IGW at altitudes of $30-40 \mathrm{~km}$ in the Earth's atmosphere. The observations provide that IGW are generated with periods of $5 \mathrm{~min}, 12-15 \mathrm{~min}$, and 23-26 min while the theory of IGW propagation in the real atmosphere predicts only a $B$-period of about $5 \mathrm{~min}$. On the other hand, the coincidence of QPV periods with those of solar oscillations (Hill et al., 1978) has already been discussed and their common genetic origin has been postulated (Galper et al., 1977) which strongly suggest the association of IGW of the Earth's atmosphere with short periodic solar oscillations. It is not yet known how the energy of solar oscillations reaches the Earth's atmosphere but it can be qualitatively postulated that the atmosphere of the Earth may experience resonant vibrations provoked by solar oscillations. These resonances can be most easily realized at radial global solar oscillations of low $l$-modes $(l=0,1,2)$. These modes of global solar oscillations with the period of about $5 \mathrm{~min}$ have been recently recorded (Grec et al., 1980; Claverie et al., 1979). Ultraviolet radiation with a wavelength ranging from 2400 to $2900 \AA$, which is known to undergo strong fluctuations (as compared with extremely weak ones in the optic rang) and is absorbed in the ozone layer, that is, practically at the same altitudes $30-40 \mathrm{~km}$, may provide the mechanism of the solar oscillation propagation. The fact that during a solar eclipse atmospheric IGW has been detected with a period of 20 min speaks in favour of this hypothesis (Goodwin and Hobson, 1978). The assumptions made need experimental verification.

\section{Acknowledgement}

We thank Dr L. P. Gorbatchev for very useful discussions.

\section{References}

Claverie, A., Isaak, G. R., McLeod, C. P., van der Raay, H. B., and Roca Cortes, T.: 1979, Nature 282, 591. Dikii, L. A.: 1969, The Theory of Vibrations of the Earth Atmosphere, Leningrad.

Galper, A. M., Kurochkin, A. V., Leikov, N. G., Luchkov, B. I., Yurkin, Yu. T.: 1974, Pribori i technika experimenta 1, 50.

Galper, A. M., Kirillov-Ugryumov, V. G., et al.: 1977, 15th Intern. Cosmic Ray Conference, Plovdiv, Vol. 4, p. 341.

Galper, A. M., Kirillov-Ugryumov, V. G., Kurochkin, A. V., Leikov, N. G., and Luchkov, B. I.: 1979, Pis'ma v JETF 30, 631 .

Galper, A. M., Kirillov-Ugryumov, V. G., Leikov, N. G., and Luchkov, B. I.: 1980, Pis'ma v JETF $31,693$.

Golitzin, G. S.: 1965, Izvestiya Akademii Nauk USSR 1, 136.

Goodwin, G. L. and Hobson, G. J.: 1978, Nature 275, 109.

Gossard, E. and Hook, U.: 1978, Waves in the Atmosphere, Moscow.

Grec, G., Fossat, E., Pomerantz, M.: 1980, Nature 288, 541.

Hill, H. A., Stebbins, R. T., and Brown, T. M.: 1978, Astrophys. J. 223, 324

Hines, C. O.: 1960, Canad. J. Phys. 38, 1441.

Kodoma, M., Sakai, T., et al.: 1975, 14th Intern. Cosmic Ray Conference, München, Vol. 3, p. 1120. 